\title{
Plataforma Móvil Basada en la Nube para Mejorar la Experiencia de Aprendizaje en la Anatomía Humana
}

\author{
Cloud-based Mobile Platform to Enhance the Learning Experience in Human Anatomy
}

Felipe Rojos ${ }^{1}$; Monica Stambuk ${ }^{1}$; Andrés Neyem ${ }^{1}$; Emilio Farfán C. ${ }^{2}$ \& Oscar Inzunza²

\begin{abstract}
ROJOS, F.; STAMBUK, M.;NEYEM, A.; FARFÁN, C. E. \& INZUNZA, O. Plataforma móvil basada en la nube para mejorar la experiencia de aprendizaje en la anatomía humana. Int. J. Morphol., 39(4):1153-1159, 2021.

RESUMEN: En la actualidad, los cursos en línea han masificado y modificado la forma en que se enseña. Tanto los MOOC como los SPOC presentan soluciones sólidas para enseñar, e incluso poseen herramientas para fomentar la colaboración. A pesar de esto, las herramientas que poseen no fomentan colaboración efectiva y tampoco tienen una forma de medirla. Por otro lado, en anatomía han surgido múltiples aplicaciones debido a las dificultades de acceso a material cadavérico, sin embargo, éstas carecen de colaboración y no entregan información enriquecida del comportamiento y aprendizaje de los estudiantes. Dado esto, presentamos una plataforma móvil basada en la nube, como estrategia de herramienta educacional, que busca fomentar la colaboración en la enseñanza para estudiantes de anatomía y entregar datos que permiten analizar y mejorar la experiencia de aprendizaje. Esta solución se desarrolló usando como eje central las metodologías ágiles de desarrollo. Para el experimento, 29 voluntarios formaron parte del grupo experimental y otros 99 del grupo de control. Se realizaron 2 pruebas para medir sus conocimientos en áreas específicas de la anatomía. Se obtuvo aumentos de 0,59 $\%$ y 2,98 \% en el puntaje de las pruebas, además, hubo una disminución en la desviación estándar de 11,434 a 5,216 en la primera prueba, y de 6,623 a 3,514 en la segunda prueba, mostrando mejora en ambos casos para el grupo experimental. Los resultados obtenidos muestran un potencial de mejorar la experiencia de aprendizaje al usar este tipo de herramientas educativas.
\end{abstract}

KEY WORDS: Aprendiendo; Aplicación; Anatomía; Educación Médica.

\section{INTRODUCCIÓN}

En los últimos años, plataformas como Coursera, Udemy, edX y otras han ofrecido MOOC (Massive Open Online Courses) o cursos públicos masivos online a miles de estudiantes. Algunas universidades han adoptado el formato MOOC a modo de suplementar sus metodologías de enseñanza. Por otro lado, los SPOCs (Small Private Online Courses) son otra solución de cursos online, pero cerrada y limitada en acceso y número de participantes. Éstos usan la misma tecnología y plataformas que los MOOCs pero orientados a ambientes de enseñanza más tradicionales (Jong, 2016). En general, las tecnologías MOOC y SPOC están diseñadas para un número variable de estudiantes, por lo que escalan sus capacidades técnicas acorde a ese número para proveer una plataforma estable y, soportar una gran cantidad de visitas y datos (Fox et al., 2014). Sin embargo, estas plataformas presentan dificultades al momento de querer desarrollar y medir actividades colaborativas.

El área de ciencias de la computación CSCL (Computer-Supported Collaborative Learning) investiga cómo solucionar los problemas de colaboración usando herramientas digitales, como aplicaciones móviles. Estas herramientas facilitan la comunicación grupal entre los estudiantes con el uso de canales síncronos, asíncronos y a distancia para lograr aprendizajes colaborativos más efectivos. Actualmente, tanto MOOCs como SPOCs tienen funcionalidades que permiten colaboración e interacción entre estudiantes, como foros y revisión de pares, pero estas herramientas no son suficientes para generar un mayor aprendizaje colaborativo efectivo (Fox et al; Manathunga \& Hernández-Leo, 2015) debido a que carecen de elementos de registro y análisis durante el proceso de aprendizaje (Zheng et al., 2014).

Se ha demostrado un gran interés por desarrollar aplicaciones móviles para la enseñanza de anatomía. Schrempf (2020) propone una aplicación móvil enfocada a un público general para que cualquiera pueda aprender de anatomía por medio de modelos 3D con realidad aumentada. De manera similar, Maric et al. (2020) presentó una aplicación para

\footnotetext{
${ }^{1}$ Departamento de Ciencias de la Computación, Escuela de Ingeniería, Pontificia Universidad Católica de Chile, Santiago, Chile.

${ }^{2}$ Departamento de Anatomía, Escuela de Medicina, Pontificia Universidad Católica de Chile, Santiago, Chile.
} 
estudiar anatomía canina utilizando modelos 3D de cráneos. Por otro lado, Dua et al. (2020) estudiaron los efectos de una aplicación que utiliza imágenes cadavéricas para la enseñanza de anatomía en estudiantes de primer año. Estas investigaciones abordan un amplio espectro de metodologías. Sin embargo, los aportes más significativos son solo modelos o aplicaciones que están en fases de prototipado, los cuales no permiten la experimentación con herramientas de CSCL. Por ello, es necesario el desarrollo de una herramienta que permita medir de forma cuantitativa el aprendizaje de los estudiantes haciendo uso de estas tecnologías.

En esta investigación se desarrolló una plataforma móvil basada en la nube para la enseñanza de anatomía a estudiantes de medicina. El objetivo de esta solución de software es generar un ambiente en donde se incentive el trabajo en equipo y se logre un aprendizaje colaborativo efectivo. Para esto, la plataforma de software educativa fue propuesta para su uso en un curso de Morfología de la Pontificia Universidad Católica de Chile. Este curso constó de 128 estudiantes, donde 29 voluntarios formaron el grupo experimental y los 99 restantes formaron el grupo de control.

\section{MATERIAL Y MÉTODO}

Metodología de desarrollo. La metodología de desarrollo de la plataforma de software educativa se realizó siguiendo los principios de las metodologías ágiles de desarrollo (Shore \& Warden, 2007). La base de esta metodología subyace en una instancia inicial de definición de requerimientos que debe tener la solución, los cuales surgen del problema que se requiera resolver, esto es usado como entrada por un proceso iterativo que se divide en 3 etapas como se señala en la Figura 1. La etapa de diseño corresponde a la etapa inicial donde se toman los requerimientos y se interpretan desde una mirada técnica para poder ser implementados. La etapa de desarrollo consiste en implementar todos los requerimientos ya analizados de la etapa anterior. Posteriormente en la etapa de test, se prueba que el desarrollo de funcionalidades

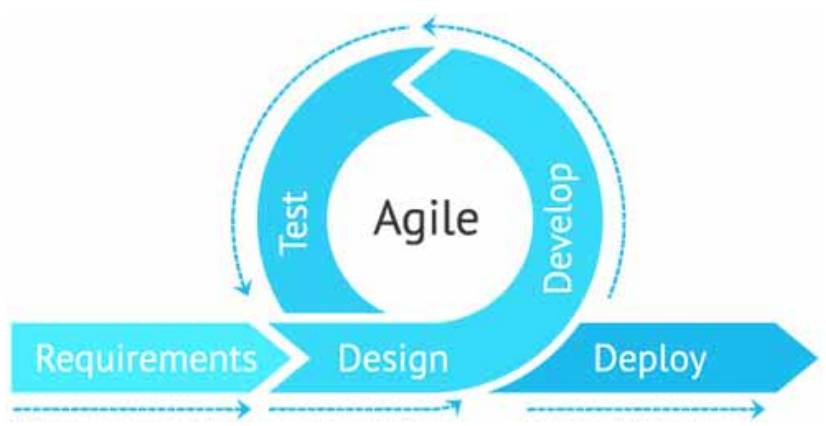

Fig. 1. Etapas de desarrollo ágil. sea el esperado, previamente definido por la etapa de requerimientos. Este ciclo se repite considerando nuevos requerimientos deseados a agregar. Una vez terminadas estas iteraciones, se procedió a realizar la etapa de despliegue, en la cual se entregaron las funcionalidades desarrolladas a una fase de completada o productiva, las cuales son utilizadas por el usuario final. Este proceso es iterado dependiendo de la llegada de nuevas funcionalidades.

El desarrollo de la plataforma de software educativa implicó varios ciclos de retroalimentación de varios semestres, en el cual participaron conjuntamente estudiantes de pregrado, postgrado y profesores pertenecientes al Departamento de Ciencia de la Computación de la Escuela de Ingeniería y al Departamento de Anatomía de la Escuela de Medicina de la Pontificia Universidad Católica de Chile.

Arquitectura. La arquitectura del ecosistema planteado está basada en el modelo arquitectónico de microservicios. Este modelo fue elegido por permitir un fácil escalamiento a la hora de realizar pruebas con grandes cantidades de usuarios y debido a su alto nivel de atomización que facilita el desarrollo y soporte informático (Newman, 2015). Esta posee tres componentes arquitectónicos: una aplicación móvil, una aplicación web y una API (Application Programming Interface) para todo el manejo inteligente de la capa de datos. El diagrama general de esta arquitectura se puede ver en la Figura 2.

JavaScript fue utilizado como único lenguaje de programación para el desarrollo de todos los componentes arquitectónicos facilitando así el soporte informático de la aplicación. Para el desarrollo móvil, se utilizó React-Native cómo framework, dado que permite el desarrollo tanto de IOS como Android con un solo código base. Por otra parte, para la aplicación web, se utilizó la librería React en conjunto con el frameworkReact-Admin para poder realizar un panel administrativo. React y React-Native tienen en común su código base, por lo que se programan de la misma forma.

En la API se utilizó el frameworkLoopback 4, el cual facilita la construcción de API Rest usando la especificación Swagger 3 y cuenta con respaldo de IBM. Ésta implementa la comunicación con una base de datos relacional SQL, las notificaciones push, registro y análisis de eventos, y formación automática de grupos.

Finalmente, la base de datos almacena las abstracciones para cada modelo de datos, en especial, el modelo de usuarios con sus respectivos roles (administrador, investigador, profesor, estudiante), modelo de evaluaciones y modelo de preguntas. Esto permite al sistema guardar toda la información necesaria para el funcionamiento de la página web y la aplicación móvil. 


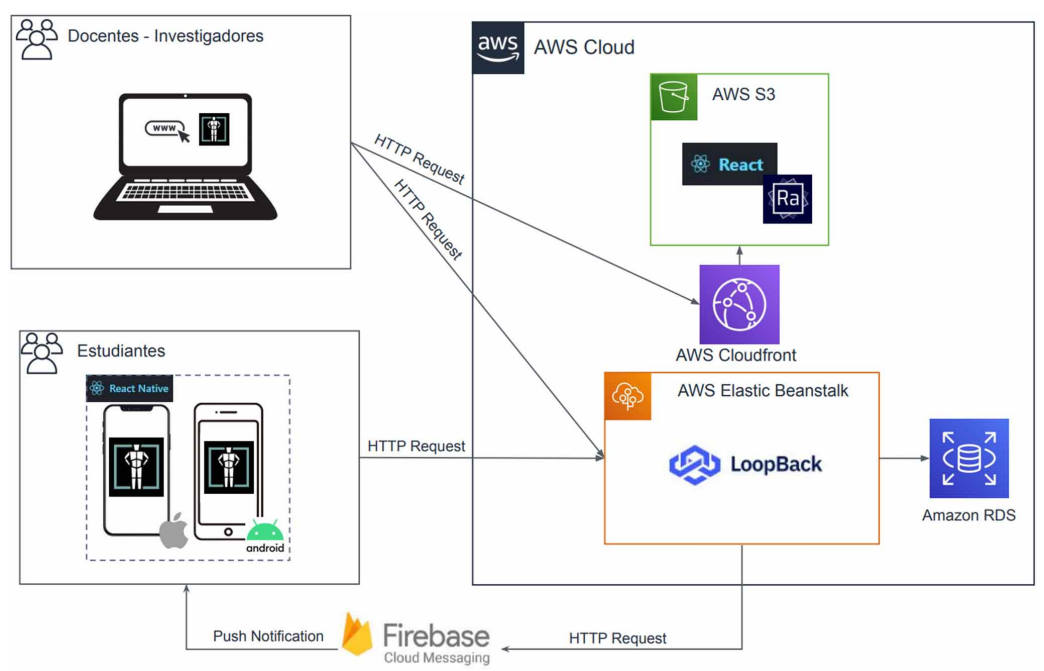

Fig. 2. Arquitectura general del sistema.

Aplicación móvil. En la aplicación móvil de la plataforma de software educativa se recrea una yincana anatómica en la cual los estudiantes deben superar desafíos acerca de diferentes tópicos de anatomía. Dentro de la aplicación los usuarios ingresan con su perfil de estudiante y realizan las actividades en conjunto con su grupo. Para colaborar entre ellos, los usuarios cuentan con un sistema de chat interno donde pueden compartir acerca de su progreso en las actividades. Cada nivel de la aplicación consiste en una serie de preguntas de anatomía, similares a las presentes en una yincana anatómica de su carrera estudiantil. Estas preguntas pueden estar acompañadas de un recurso visual, ya sea imagen o modelo 3D. En la Figura 3 se presentan las principales vistas en donde los usuarios realizan estas acciones. Adicionalmente, la interfaz de navegación de componentes 3D corresponde a una nueva versión del componente desarrollado en la herramienta educativa Anatomicis (Inzunza et al., 2017). En esta, los estudiantes pueden rotar y agrandar el modelo para analizarlo en profundidad. Estos componentes cuentan con la capacidad de almacenar data enriquecida en forma de puntos informativos y con ello complementar el análisis de los estudiantes.

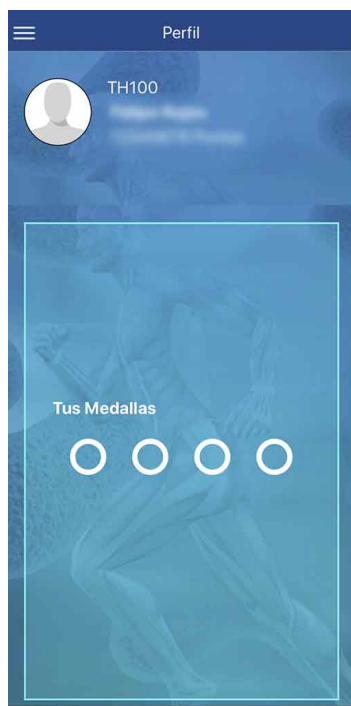

A

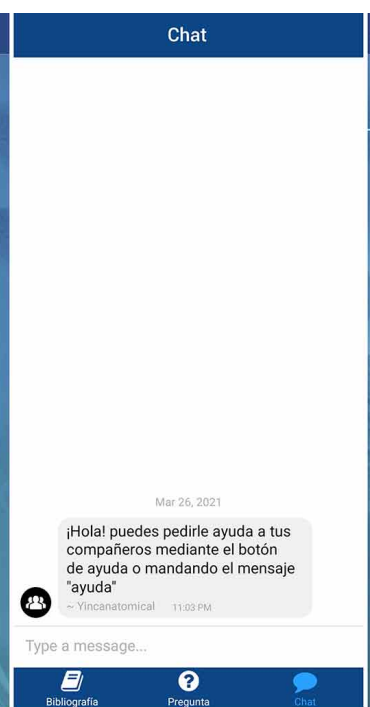

B

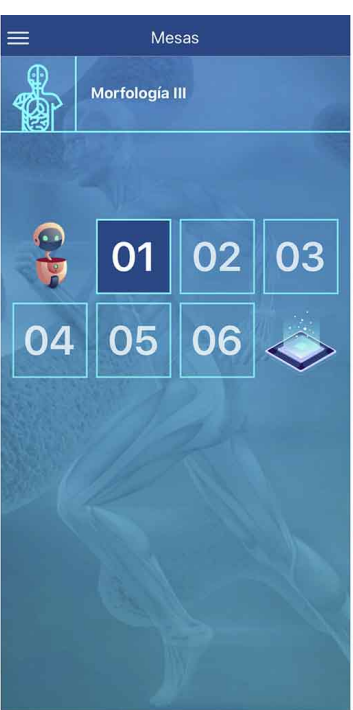

C

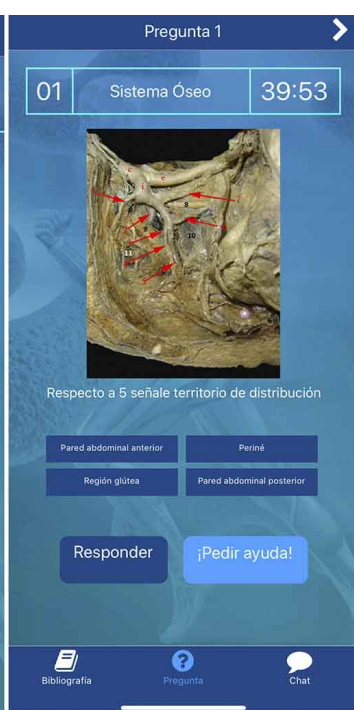

D

Fig. 3. Diseño del aplicativo. En a) se presenta el perfil de un usuario, el cual muestra sus estadísticas y logros obtenidos hasta el momento. Aquí el usuario puede cambiar parámetros como su nombre y grupo al que pertenece. En b) se ve el chat con el que puede interactuar el equipo de trabajo para realizar las actividades grupales. En c) se muestra la cadena de niveles que el estudiante debe superar para completar la actividad; en esta se utilizan diferentes colores para diferenciar aquellos que ya han sido superados de los que están pendiente. El usuario puede seleccionar y repetir cualquier nivel que ya haya superado previamente por medio de esta vista. En d) se despliega la interfaz de selección de alternativas para contestar las preguntas de cada nivel, las cuales están acompañadas de una imagen o un modelo 3D. 
Por último, en la Figura 4 se muestra un tipo de pregunta especial de alta dificultad, la pregunta infarto, que tiene como objetivo incentivar la colaboración entre los miembros del equipo. Para lograr esto, dicha pregunta se presenta de manera simultánea ante todo el grupo, el cual debe coordinar mediante la funcionalidad de pedir ayuda, que dispone de un chat para coordinar la respuesta, preguntar por dudas y responder estas dudas.

Versión Web. La versión web de la plataforma de software educativa está orientada a docentes e investigadores, por lo que cumple un rol principalmente de administración y recolección de datos. Ésta permite crear y editar manualmente las preguntas, los grupos y las evaluaciones para poder generar las intervenciones con los estudiantes. A su vez, cuenta con la funcionalidad de exportar e importar datos en formato .csv, para facilitar su análisis con otras plataformas.

En la Figura 5 se exhibe el panel de administración de cursos, en esta se puede ver un listado de tres cursos de anatomía con sus datos principales. En esta interfaz es posible crear, editar y eliminar cursos, además de poder exportar o importar información relacionada a los cursos. Por medio del menú lateral izquierdo, se puede navegar dentro de los otros paneles, como el de grupos y evaluaciones, para administración de su contenido.

PAUTA ÉTICA. Los estudiantes firmaron un consentimiento informado en el cual se puntualizó que su participación en este estudio era completamente voluntaria. Este consentimiento fue presentado por medio de un formulario en Google Form, en el cual se declara que solo se usarían datos relacionados a métricas de uso de la aplicación y su desempeño académico en el curso.

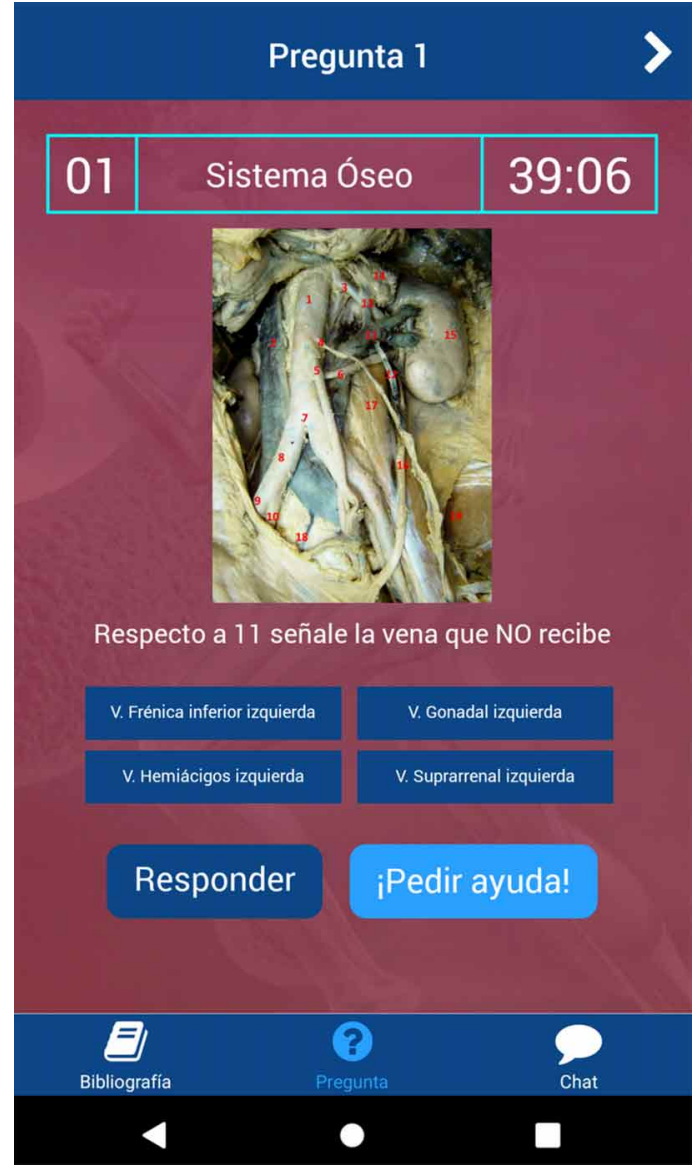

Fig. 4. Pregunta Infarto.

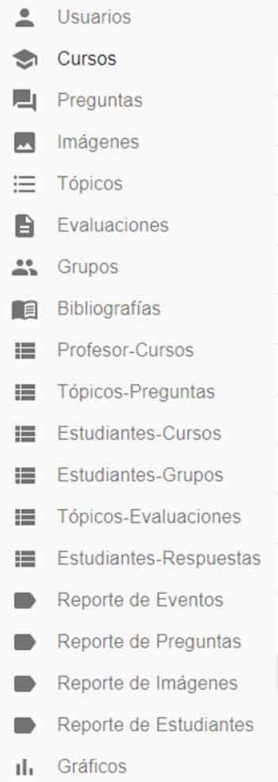

Fig. 5. Panel de administración. 


\section{RESULTADOS}

En la primera prueba, que contaba con un puntaje máximo de 100 puntos, el grupo control obtuvo un promedio de 90,53 puntos y el grupo experimental obtuvo 91,07 puntos (Tabla I), lo que se traduce en una mejora del 0,59 $\%$. A su vez, la desviación estándar en esta prueba del grupo de control es de 11,43 y del grupo experimental de 5,22, lo que demuestra una mayor consistencia en el grupo experimental. En la segunda prueba, que contaba con un máximo de 56 puntos, el grupo de control obtuvo un promedio de 49,41 puntos y el grupo experimental de 50,93 puntos, lo que corresponde a una mejora de $2,98 \%$. Similar a la primera prueba, la desviación estándar del grupo experimental mejora su consistencia al obtener un valor de 3.514 y en el grupo de control con un valor de 6,62 (Tabla II).

Tabla I. Notas del grupo control vs grupo experimental en examen 1.

\begin{tabular}{lccc}
\hline Grupos & N $^{\text {o }}$ & Promedio & D. E. \\
\hline Grupo Control & 99 & 90,529 & 11,434 \\
Grupo Experimental & 29 & 91,069 & 5,216 \\
\hline
\end{tabular}

A lo largo del experimento, hubo un total de 12 estudiantes que dejaron la actividad sin completar (drop outs), con lo que 17 completaron todos los niveles. Con respecto al uso de la aplicación, los tiempos de uso en el nivel 1 son irregulares al haber usuarios que superan los 55 minutos dentro de la etapa, lo que luego se estabiliza a partir del nivel 2 con medias entre los 2 y 5 minutos. En promedio, los usuarios invirtieron 14 minutos y medio en completar toda la actividad, por lo que su velocidad de respuesta estuvo alrededor de los 35,98 segundos/respuesta. En este ítem, el usuario más rápido tuvo una velocidad de 24,77 segundos/respuesta mientras que el más lento necesitó de 119,33 segundos/respuesta.

Tabla II. Notas del grupo control vs grupo experimental en examen 2

\begin{tabular}{lccc}
\hline Grupos & $\mathrm{N}^{\mathrm{o}}$ & Promedio & $\mathrm{D} . \mathrm{E}$. \\
\hline Grupo Control & 99 & 49,412 & 6,623 \\
Grupo Experimental & 29 & 50,931 & 3,514 \\
\hline
\end{tabular}

\section{DISCUSIÓN}

El aprendizaje colaborativo promueve el debate y la discusión, lo que potencia las habilidades argumentativas del estudiante y su criterio para tomar decisiones, además de promover el liderazgo, y con ello las habilidades sociocognitivas del estudiante para dar relevancia a la interacción social y comunicación (Lizcano-Dallos et al., 2019). En la carrera de medicina es necesario desarrollar estas habilidades socio-cognitivas de manera activa, ya que algunas de estas no se forman con los años de trabajo en clínica y son necesarias al momento de tratar con pacientes (Aspegren \& Lønberg-Madsen, 2005). El desarrollo temprano de estas habilidades argumentativas constituye una herramienta formativa relevante que permite superar las diferencias de origen de los estudiantes de las carreras de la salud.

Vivian et al. (2016) muestra cómo la resolución de problemas en equipo fomenta el aprendizaje colaborativo. Para esto, se menciona el fomentar que los estudiantes pregunten sus dudas y respondan las de sus compañeros. En este trabajo, se abordó la resolución de problemas en equipo con la instancia de la pregunta infarto, además de la opción de pedir ayuda en cada problema. Con ello, los estudiantes podían preguntar a sus compañeros por medio del chat acerca de las preguntas que no lograban resolver y, en el caso de la pregunta infarto, se veían obligados a coordinar sus respuestas.

Por otro lado, Johnson \& Johnson (1999) y BlancoIzquierdo et al. (2016), proponen los siguientes aspectos como fundamentales para ofrecer un ambiente efectivo para el aprendizaje colaborativo: (1) Un objetivo en común para los participantes del curso. (2) Interacción y comunicación entre cada participante. (3) Responsabilidades individuales para cada participante. (4) Promover las habilidades sociales, tales como el liderazgo o la capacidad de toma de decisiones. La aplicación desarrollada en este trabajo aborda estos puntos con: (1) presentar como objetivo el completar todas las estaciones anatómicas; (2) permitir la comunicación e interacción mediante la funcionalidad de ayuda y la pregunta infarto; (3) estimular el superar una estación para que el resto del equipo pueda avanzar a la siguiente estación; (4) ofrecer un espacio de discusión para encontrar la solución a la pregunta infarto.

Lo anterior se ve reflejado en mejoras cuantitativas en el desempeño de los estudiantes. Sin embargo, no observamos las mejoras esperadas en el rendimiento. Uno de los aspectos fundamentales que influyó en el cuasi-experimento fue la pandemia, que afectó la coordinación y compromiso de los estudiantes. Además, la pandemia dificultó el proceso de incorporación a la plataforma. Si bien en este cuasi experimento se utilizó las herramientas oficiales para fases experimentales de aplicaciones móviles, es decir Testflight para iOS y un archivo instalable para Android, no se logró dar el soporte técnico en casos que fuese necesario. Dada la distancia social requerida, solo se pudo brindar ayuda a través de correos electrónicos y videotutoriales. Por esta misma razón, tampoco se pudo proveer dispositivos móviles a 
estudiantes que lo requirieran. Hynes \& Younie (2018) mencionan la importancia de contar con un sistema tecnológico regulado y probado previamente, de manera que las actividades puedan realizarse de manera efectiva. Dado esto, se sugiere simplificar el proceso de instalación, y generar otros espacios de comunicación inmediata para los estudiantes.

Por otro lado, con el objetivo de promover sus habilidades sociales, formamos los grupos de trabajo de forma aleatoria, es decir, generamos un espacio en el que los integrantes no necesariamente se conocían entre sí. Esto no tuvo una buena recepción lo que se ve reflejado en que algunos grupos no pudieran superar el primer nivel, pues era necesario que todos los integrantes completaran cada etapa de la yincana de manera individual para que el grupo pudiera continuar. Esta situación se evidencia en los resultados, donde 9 de los 29 estudiantes dejaron de usar la aplicación tras haber respondido el primer nivel. Estos resultados coinciden con lo presentado por Ku et al. (2013) acerca del impacto de las relaciones personales previas en el compromiso de los participantes y la efectividad del trabajo en equipo. Por ello, se sugiere para futuros usos del aplicativo, permitir la formación de grupos de manera voluntaria.

Respecto a la retroalimentación recibida, los estudiantes resaltaron la necesidad de transparentar el estado de los otros miembros en la pantalla de espera, además de mostrar interés en poder compartir las preguntas de la yincana con los otros miembros del grupo. En cuanto a la utilidad de la aplicación, los estudiantes apreciaron la plataforma como una buena herramienta para el estudio de anatomía de manera remota, sobre todo con el contexto de la pandemia. De manera adicional, apreciaron la flexibilidad y libertad que entrega el aplicativo para equivocarse en su modalidad de juego.

Si bien es relevante considerar que esta plataforma es de carácter complementario y no busca reemplazar la práctica en vivo con cadáveres, ésta posee potencial para promover aún más el aprendizaje colaborativo. Al Mamun et al. (2020) presentan la importancia y efectividad de usar un esquema enfocado en toma de decisiones y cuestionamiento investigativo para lograr que los estudiantes se involucren y utilicen las plataformas online de manera independiente. En esta aplicación educativa, este enfoque se puede implementar mediante la resolución de casos clínicos. Ya que se ha demostrado que estos requieren de un trabajo colaborativo, donde los profesionales contrastan sus distintos puntos de vista para llegar a un diagnóstico (Aguilera \& Quintero, 2021). Por otro lado, se ha demostrado que la colaboración fomenta el aprendizaje efectivo de los estudiantes (Zheng et $a l$.). Por ende, agregar análisis de casos fomentará el aprendizaje efectivo de los estudiantes.
La aplicación móvil permitió a los usuarios realizar la experiencia de una yincana virtual y recopilar información en tiempo real acerca de sus acciones dentro de ésta. Así, fue posible realizar análisis cuantitativos acerca del comportamiento de los usuarios. Si bien se ha demostrado la importancia de este tipo de aplicaciones en el área de anatomía (Lozano et al., 2018), para este tipo de intervenciones se requiere compromiso de los participantes y no resulta conveniente dejarlo como algo optativo.

\section{CONCLUSIONES}

Esta plataforma de software educativo es una herramienta que genera valor en el proceso de estudio de los alumnos, tanto por el material presentado en este como por la flexibilidad que da a los estudiantes para practicar y equivocarse en sus desafíos. A su vez, se logró recolectar datos con el fin de conocer el comportamiento de los estudiantes.

Si bien la pandemia afectó los resultados esperados, su naturaleza grupal promueve la competitividad de los participantes, lo que hace que los estudiantes salgan de su zona de confort y ejerciten contenidos que no son de su conocimiento.

Por último, esta plataforma posee las bases necesarias para potenciar el estudio de la anatomía de forma remota y con los cambios sugeridos permitirá fomentar aún más el aprendizaje colaborativo efectivo.

\section{AGRADECIMIENTOS}

Este estudio fue financiado por la beca Conicyt N. 21200443. Quisiéramos agradecer a los estudiantes de anatomía de la Pontificia Universidad Católica de Chile que participaron de este proyecto.

ROJOS, F.; STAMBUK, M.;NEYEM, A.; FARFÁN, C. E. \& INZUNZA, O. Cloud-based mobile platform to enhance the learning experience in human anatomy. Int. J. Morphol., 39(4):1153-1159, 2021.

SUMMARY: Online courses have become popular nowadays, changing the way different subjects are taught. Both MOOCs and SPOCs present robust solutions for teaching, and even have tools to encourage collaboration. Nevertheless, the tools used do not foster effective collaboration nor can they adequately measure outcomes. On the other hand, multiple applications have emerged in anatomy as a result of the difficulty in accessing 
cadaveric material, however, these lack the collaborative aspect, and do not provide information on student behavior and learning. Given this, we present a cloud-based mobile platform, as an educational tool strategy, to promote collaboration in teaching for anatomy students and provide data that allows to analyze and improve the learning experience. This solution was developed using agile development methodologies as the central axis. In this study, 29 volunteers were part of the experimental group and another 99 the control group; 2 tests were performed to measure knowledge in specific areas of anatomy. Increases of $0,59 \%$ and $2,98 \%$ were obtained in the test score, in addition, there was a reduction in the standard deviation from 11,434 to 5,216 in the first test, and from 6,623 to 3,514 in the second test, showing improvement in both cases for the experimental group. The results obtained indicate a potential to improve the learning experience when using this type of educational tool.

KEY WORDS: Learning; App; Anatomy; Medical Education.

\section{REFERENCIAS BIBLIOGRÁFICAS}

Aguilera, A., \& Quintero, J. A descriptive analysis for a collaborative work process: a complex real medical case in the radiological field. J. Med. Imaging Health Inform., 11(2):535-44, 2021

Al Mamun, M. A.; Lawrie, G. \& Wright, T. Instructional design of scaffolded online learning modules for self-directed and inquiry-based learning environments. Comput. Educ., 144:103695, 2020.

Aspegren, K. \& Lønberg-Madsen, P. Which basic communication skills in medicine are learnt spontaneously and which need to be taught and trained? Med. Teach., 27(6):539-43, 2005.

Blanco-Izquierdo, F.; González, C. S. \& Collazos, C. A. Modelado y Evaluación de la Interacción en el Aprendizaje CSCL y Juegos Colaborativos. 2016. Disponible en: https://www.researchgate.net/ publication/294428563_Modelado_y_Evaluacion_ de_la_Interaccion_en_el_Aprendizaje_CSCL_y_Juegos_Colaborativos

Dua, A.; Coppola, K. M.; Mulheron, G. W.; Troupe, D. \& Lebeau, R. Development of a novel peer-sharing application to supplement learning from cadaveric dissection. Anat. Sci. Educ., 2020. DOI: https:// www.doi.org/10.1002/ase.2032

Fox, A.; Patterson, D. A.; Ilson, R., Joseph, S.; Walcott-Justice, K. \& Williams, R. Software Engineering Curriculum Technology Transfer: Lessons learned from MOOCs and SPOCs. Electrical Engineering and Computer Sciences, University of California at Berkeley, Technical Report No. UCB/EECS-2014-17, 2014. Disponible en: https:// www2.eecs.berkeley.edu/Pubs/TechRpts/2014/EECS-2014-17.pdf

Hynes, P. \& Younie, S. Bring your own device? In: Younie, S. \& Bradshaw, P. (Eds.). Debates in Computing and ICT Education. Londres, Routledge, 2018. pp.153-66.

Inzunza, O.; Neyem, A.; Sanz, M. E.; Valdivia, I.; Villarroel, M.; Farfán, E.; Matte, A. \& López-Juri, P. Anatomicis Network: a cloud-based educational software platformto enhance anatomy teaching in medical education. Int. J. Morphol., 35(3):1168-77, 2017.

Johnson, D. W. \& Johnson, R. T. Making cooperative learning work. Theory Pract., 38(2):67-73, 1999.

Jong, J. P. The effect of a blended collaborative learning environment in a Small Private Online Course (SPOC): a comparison with a lecture course. J. Balt. Sci. Educ., 15(2):194-203, 2016.

Ku, H. Y.; Tseng, H. W. \& Akarasriworn, C. Collaboration factors, teamwork satisfaction, and student attitudes toward online collaborative learning. Comput. Hum. Behav., 29(3):922-9, 2013.
Lizcano-Dallos, A. R.; Vargas-Daza, M. \& Barbosa-Chacón, J. W. Collaborative learning and technologies in higher education. Characteristics, advantages and disadvantages for its implementation. Palma, EDULEARN19 Proceedings, 11th International Conference on Education and New Learning Technologies, 2019. pp.7713-9.

Lozano, M. T. U.; Ugidos, G. F.; San Pedro, A. B.; Pedro, C. B.; Manzoor, S. \& Mendez, J. A. J. The importance of the new Apps technology in the study of anatomy by the students of medicine. Salamanca, TEEM'18: Proceedings of the Sixth International Conference on Technological Ecosystems for Enhancing Multiculturality, ACM International Conference Proceeding Series, 2018. pp.362-7.

Manathunga, K. \& Hernández-Leo, D. Has research on collaborative learning technologies addressed massiveness? A literature review. $J$. Educ. Technol. Soc., 18(4):357-70, 2015.

Maric', M.; Gomerc 'ic', T.; Korpes, K.; Kolenc, M. \& ?uras, M. Digital anatomical canine anatomical skull model. Veterinar, 58(1):16-22, 2020.

Newman, S. Building Microservices: Designing Fine-Grained Systems. Sebastopol, O'Reilly Media, 2015.

Schrempf, M. T. Fantastic Voyage: An Augmented Reality Approach to Anatomical Education for the General Public. Tesis de Magister. Viena, Fakultät für Informatik der Technischen Universität Wien, 2020.

Shore, J. \& Warden, S. The Art of Agile Development: Pragmatic Guide to Agile Software Development. Newton, O'Reilly Media, 2007.

Vivian, R.; Falkner, K.; Falkner, N. \& Tarmazdi, H. A method to analyze computer science students' teamwork in online collaborative learning environments. ACM Transactions on Computing Education, 16(2):7, 2016. DOI: https://www.doi.org/10.1145/2793507

Zheng, L.; Yang, J.; Cheng, W. \& Huang, R. Emerging approaches for supporting easy, engaged and effective collaborative learning. J. King Saud Univ. Comput. Inf. Sci., 26(1):11-6, 2014.

Dirección para correspondencia:

Prof. Felipe Rojos Almuna

Departamento de Ciencias de la Computación

Escuela de Ingeniería

Pontificia Universidad Católica de Chile

Av. Libertador Bernardo O'Higgins \#340

Santiago

CHILE

E-mail: farojos@uc.cl

ORCID: 0000-0002-9016-1259

ORCID: 0000-0002-8819-2945

Recibido : 30-04-2021

Aceptado: 27-05-2021 